\title{
EchoGéo
}

$11 \mid 2010$

Madagascar

\section{Interview de Jean-Pierre Palisse}

\section{Alexis Sierra}

\section{OpenEdition}

Journals

Édition électronique

URL : https://journals.openedition.org/echogeo/11681

DOI : 10.4000/echogeo.11681

ISSN : 1963-1197

\section{Éditeur}

Pôle de recherche pour l'organisation et la diffusion de l'information géographique (CNRS UMR 8586)

\section{Référence électronique}

Alexis Sierra, «Interview de Jean-Pierre Palisse », EchoGéo [En ligne], 11 | 2010, mis en ligne le 24 février 2010, consulté le 31 juillet 2021. URL : http://journals.openedition.org/echogeo/11681 ; DOI : https://doi.org/10.4000/echogeo.11681

Ce document a été généré automatiquement le 31 juillet 2021.

EchoGéo est mis à disposition selon les termes de la licence Creative Commons Attribution - Pas d'Utilisation Commerciale - Pas de Modification 4.0 International (CC BY-NC-ND) 


\title{
Interview de Jean-Pierre Palisse
}

\author{
Alexis Sierra
}

1 Jean-Pierre Palisse est Directeur Général Adjoint de l'IAU IdF (Institut d'Aménagement et d'Urbanisme île de France), Directeur du Département Urbanisme, Aménagement et Territoires

2 - Alexis Sierra (AS). L'Institut d'Aménagement et d'Urbanisme de la Région Ile-deFrance emploie-t-il des géographes ? Combien?

3 Jean-Pierre Palisse (JPP). L'IAU emploie en tant qu'urbanistes-chargés d'études, une vingtaine de géographes ayant pour la plupart suivi une formation complémentaire en matière d'aménagement urbain ou rural. De plus il emploie, sous l'appellation " géomaticien », une vingtaine de géographes ayant suivi une formation spécialisée en informatique dans le domaine de la cartographie et des SIG. Ces géomaticiens sont répartis dans lesdifférents départements de l'IAU.

4 - AS. Quelles sont les compétences géographiques les plus sollicitées : un savoir-faire sur les outils (cartographie, géomatique) ou l'analyse géographique?

5 - JPP. Ces deux compétences sont sollicitées. Les chargés d'études sont amenés à pratiquer la cartographie, l'approche quantitative et l'analyse géographique entendue comme l'intégration des différentes thématiques de la géographie (physique, paysagère et culturelle, économique, sociale, géopolitique) associée à une lecture à différentes échelles et permettant notamment de construire un discours territorial.

6 Pour les géomaticiens, c'est évidemment leur savoir-faire sur les outils qui est le plus sollicité au travers de la cartographie, du croisement des données et de l'analyse spatiale permettant le développement des SIG.

7 - AS. L'analyse géographique relève-t-elle avant tout de l'analyse spatiale quantitative? Aborde-t-elle également l'analyse :

- des représentations sociales de l'espace régional?

- des dimensions politiques et géopolitiques?

- des dimensions culturelles?

8 - JPP. L'analyse spatiale quantitative dont les SIG sont les principaux outils est le socle permettant aux chargés d'études de développer l'analyse des représentations et des 
dimensions socio-économiques, culturelles et politiques. Ces travaux ont aussi une visée évaluative, prospective et stratégique. Le savoir-faire cartographique des géographes est aussi mobilisé pour l'illustration et la communication d'informations et de messages spatialisés.

9 - AS. Quelles sont les relations avec les institutions de recherche qui produisent un savoir géographique (Universités, Etablissements Publics Scientifiques et Techniques, laboratoires privés)?

10 - JPP. Ces relations sont constantes dans le cadre de nos travaux d'études ou de notre participation à des événements (séminaires, colloques, table-rondes...). L'IAU s'associe parfois à des travaux de recherche menés par des laboratoires universitaires. Des conventions de partenariat sont passées avec des universités pour des études ou des formations (Institut Français d'Urbanisme par exemple) ainsi qu'avec des organismes publics comme l'IGN. Par ailleurs, l'IAU accueille de nombreux stagiaires en provenance des différents cycles de formation en aménagement.

11 - AS.L'IAU possède un « pool » cartographique reconnu. Fonctionne-t-il par commande de la Région exclusivement?

12 - JPP. Fondation d'utilité publique, l'IAU conduit l'essentiel de ses travaux dans le cadre du programme mutualisé fixé par son conseil d'administration. C'est dans ce cadre qu'est réalisé l'essentiel de son travail cartographique. La Région, qui est son principal financeur, est naturellement le principal bénéficiaire de ces travaux mais n'en est pas le commanditaire exclusif. Les commandes directes de cartes originales de la part la Région sont relativement exceptionnelles. L'IAU conduit également des études dans un cadre commercial pouvant conduire à la production de cartographie adaptée aux commandes particulières des contractants.

13 - AS. Quelles sont les priorités thématiques de la production cartographique de l'IAU ?

14 - JPP. La production cartographique de l'IAU est aussi variée que ses domaines d'études: aménagement urbain et rural, économie, démographie, habitat et équipements, transport, environnement dans toutes ses dimensions. Elle concerne donc à tous les thèmes relevant de l'aménagement et du développement régional. Le système d'information géographique régional développé et géré par l'IAU a pour principal intérêt de permettre de croiser toutes ces thématiques favorisant ainsi les démarches holistiques attendues dans les approches de développement durable.

15 - AS. Quels sont les supports de la production cartographique (atlas papier, atlas numérique, site web, rapport pour la Région,...) (hiérarchiser le cas échéant)?

16 - JPP. La production cartographique de l'IAU utilise toutes sortes de support en fonction de ses cibles. L'IAU poursuit la production traditionnelle d'études ou d'atlas papier (Atlas rural, Atlas de franciliens, Atlas de la santé, etc.) et de publications périodiques ( les Cahiers », « note rapide ») qui sont largement illustrées de cartes. Parallèlement le site Web de l'IAU propose plusieurs centaines de cartes interactives sur l'Ile-deFrance, une centaine en accès libre, les autres en accès réservés sur abonnement.

17 - AS. Cette production cartographique sert-elle en premier lieu la politique de communication régionale?

18 - JPP. Non, absolument pas. Elle sert d'abord à réaliser et à valoriser les travaux de l'IAU et à fournir une information lisible, objective et fiable sur les différents aspects de l'Ilede-France. Ces productions sont, bien entendu, utilisées par la Région pour ses travaux 
et parfois pour sa communication mais elles le sont aussi par de nombreux organismes ou médias s'intéressant aux problématiques régionales.

19 - AS. Le savoir-faire géographique de l'IAU est-il facilement reconnu et utilisé par les élus et administrateurs régionaux, je pense en particulier à toutes les démarches qui font appel à l'emboîtement des échelles ?

20 - JPP. Il faudrait évidemment leur poser la question directement. Si l'on en croit les déclarations des membres de notre conseil d'administration et notamment de son Président et si l'on en juge par les demandes formulées par les élus, le plus souvent par le canal de leurs services, il semble que la compétence cartographique de l'IAU soit largement reconnue au point d'occulter parfois, comme l'arbre cache la forêt, son savoir-faire en matière d'analyse et sa capacité de proposition.

21 - AS. La production de l'IAU est-elle également diffusée vers le grand public et comment ?

22 - JPP. Oui, l'IAU s'attache à faire connaître largement les résultats de ses observations et de ses analyses afin d'éclairer le débat sur l'aménagement régional. Il a renforcé son effort de diffusion vers le grand public notamment au moyen de son site internet (www.iau-idf.fr) récemment refondu. Grâce à ce site, la plupart de ses travaux, documents, études, Cahiers, Note rapide, Newsletter, cartes interactives, sont consultables intégralement et gratuitement.

23 L'IAU publie plusieurs périodiques :

- Les Cahiers, revue trimestrielle tirée de 3000 à 5000 exemplaires à destination d'élus et d'experts de l'aménagement

- Une quarantaine par an de "note rapide ", 4 pages à destination du même public

- La Newsletter, lettre d'information mensuelle sur le web à destination du grand public.

24 Par ailleurs les agents de l'IAU font fréquemment connaître ses travaux à l'occasion de leurs interventions dans le cadre d'événements, colloques ou séminaires, ou d'interventions directes dans les médias (journaux, radio, télévision). 